\title{
Hydrodynamic Modeling of AlGaN/GaN HEMTs
}

\author{
S. Vitanov, V. Palankovski, S. Murad ${ }^{\dagger}$, T. Rödle ${ }^{\dagger}$, R. Quay*, and S. Selberherr* \\ Advanced Materials and Device Analysis Group at * \\ *Institute for Microelectronics, TU Vienna, A-1040 Vienna, Austria \\ \{vitanov|palankovski|selberherr\}@iue.tuwien.ac.at \\ ${ }^{\dagger}$ ICRF, NXP Semiconductors, 6534AE, Nijmegen, The Netherlands \\ \{ saad.murad|thomas.roedle\}@nxp.com \\ ¥ Fraunhofer Inst. for Solid-State Physics (IAF), D-79108 Freiburg, Germany \\ ruediger.quay@iaf.fraunhofer.de
}

\begin{abstract}
For the needs of high electron mobility transistors (HEMTs) optimization a reliable software simulation tool is required. Due to the high electric field in the device channel a hydrodynamic approach is used to properly model the electron transport. We modify an existing hydrodynamic mobility model in order to achieve a better agreement with Monte Carlo (MC) simulation data and measured DC and AC characteristics of AlGaN/GaN HEMTs.
\end{abstract}

\section{Introduction}

Wide bandgap GaN-based HEMTs exhibit properties which make them eligible for the use in high-power radio frequency applications. To further optimize and down-scale the structures a reliable simulation tool is needed. Our two-dimensional device simulator Minimos-NT [1] has been extended for the GaN-material system [2]. In this work the carrier transport modeling is presented together with calibration to experimental data.

\section{Mobility Model}

Since the longitudinal electric field in the channel reaches peak values of above 500 $\mathrm{kV} / \mathrm{cm}$, a hydrodynamic approach is required to properly model electron transport and energy relaxation. The low-field electron mobility $\mu^{\mathrm{LI}}$ is modeled using the expression proposed by Caughey and Thomas [3] with coefficients which depend on the lattice temperature $T_{\mathrm{L}}$ :

$$
\begin{aligned}
& \mu^{\mathrm{LI}}=\mu^{\mathrm{min}}+\frac{\mu^{\mathrm{L}}-\mu^{\mathrm{min}}}{1+\left(C_{\mathrm{I}} / C^{\mathrm{ref}}\right)^{\gamma_{0}}}, \text { where } \\
& \mu^{\mathrm{L}}=\mu_{300}^{\mathrm{L}}\left(\frac{T_{\mathrm{L}}}{300 \mathrm{~K}}\right)^{\gamma_{1}}, \mu^{\min }=\mu_{300}^{\min }\left(\frac{T_{\mathrm{L}}}{300 \mathrm{~K}}\right)^{\gamma_{1}}, \text { and } C^{\mathrm{ref}}=C_{300}^{\text {ref }}\left(\frac{T_{\mathrm{L}}}{300 \mathrm{~K}}\right)^{\gamma_{2}} .
\end{aligned}
$$

$\mathrm{C}_{\mathrm{I}}$ denotes the concentration of ionized impurities. Fig. 1 and Fig. 2 compare the model to own MC simulation results and experimental data as a function of concentration and lattice temperature, respectively. The model is calibrated against the MC data, which 


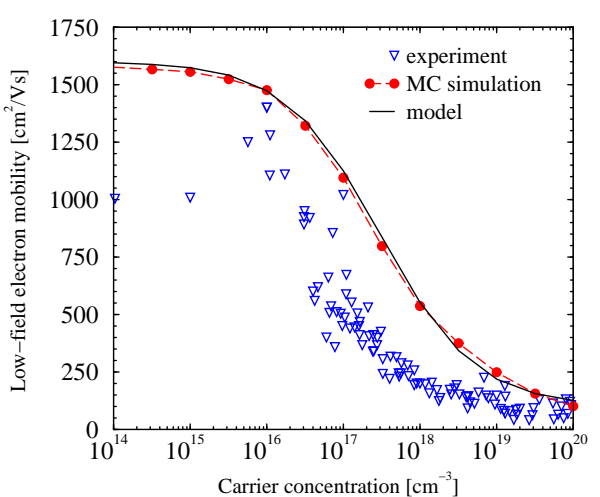

Figure 1: Mobility vs. concentration at $300 \mathrm{~K}$ : model comparison to Monte Carlo and experimental data.

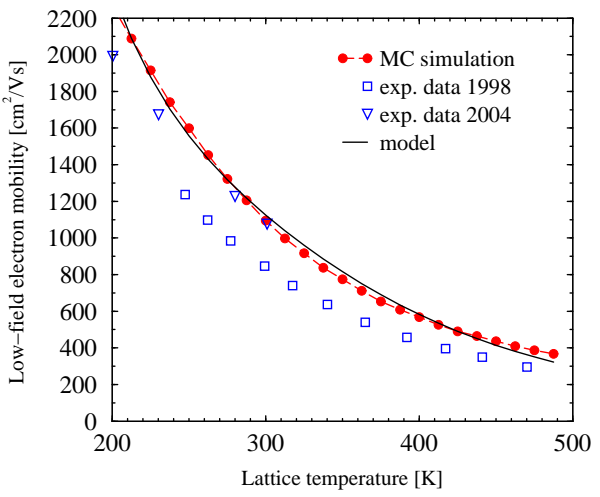

Figure 2: Low-field electron mobility as a function of lattice temperature at carrier concentration of $10^{17} \mathrm{~cm}^{-3}$.

exhibits a mobility slightly higher than suggested by most experimental results. The latter show increasing values in recent years, due to the improved quality of the material samples. The calibrated model parameter values are listed below:

\begin{tabular}{cccccc}
\hline$\mu_{300}^{\mathrm{L}}$ & $\mu_{300}^{\min }$ & $C_{300}^{\mathrm{ref}}$ & $\gamma_{0}$ & $\gamma_{1}$ & $\gamma_{2}$ \\
\hline $1600 \mathrm{~cm}^{2} / \mathrm{Vs}$ & $100 \mathrm{~cm}^{2} / \mathrm{Vs}$ & $3 \times 10^{17} \mathrm{~cm}^{-3}$ & 0.7 & -3.0 & 4.4 \\
\hline
\end{tabular}

The hydrodynamic mobility model proposed by Hänsch [4] has been modified to account for GaN specific effects:

$$
\mu^{\mathrm{LIT}}=\frac{\mu^{\mathrm{LI}}\left(T_{\mathrm{n}} / T_{\mathrm{L}}\right)^{\gamma}}{\left(1+\alpha^{1 / \beta}\left(T_{\mathrm{n}}-T_{\mathrm{L}}\right)^{1 / \beta}\right)^{\beta}}, \text { where } \alpha=\frac{3 \mathrm{k}_{\mathrm{B}} \mu^{\mathrm{LI}}}{2 \mathrm{q} \tau_{\varepsilon}\left(v_{\mathrm{sat}}\right)^{2}} .
$$

$T_{\mathrm{n}}$ is the electron temperature, $\tau_{\varepsilon}$ the energy-dependent relaxation times [2], and $v_{\text {sat }}$ the saturation velocity. The latter is modeled with temperature dependence as given in [5]. The parameter $\beta$ is used to model the mobility for moderate carrier temperatures. The parameter $\gamma$ models the decay of the electron velocity in $\mathrm{GaN}$ and $\mathrm{AlGaN}$ at high electron temperatures. The conventional Hänsch model corresponds to the $\beta=1, \gamma=0$ parameter set.

\section{Simulated Device Structure}

Fig. 3 shows a schematic of the investigated device featuring a field-plate. The gate length $1_{\mathrm{g}}=0.3 \mu \mathrm{m}$ and the field-plate extension length $l_{\mathrm{FP}}=l_{\mathrm{g}}$. All layers are nonintentionally doped except the supply layer, which provides additional carriers in the channel and improves the access resistance. The polarization charge density at the $\mathrm{Al}_{0 .}{ }_{3} \mathrm{Ga}_{0.7} \mathrm{~N} / \mathrm{GaN}$ heterointerface is found to be $\approx 1.1 \times 10^{13} \mathrm{~cm}^{-2}$ from the DC transfer characteristics. The positive charge at the channel/spacer interface is compensated by a commensurate negative surface charge at the barrier/cap interface. 


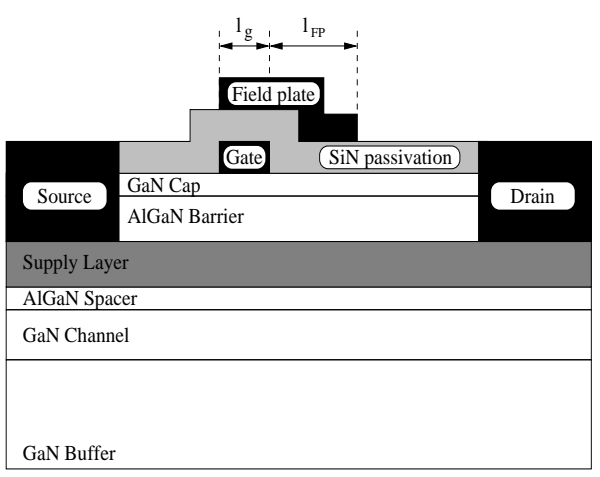

Figure 3: Schematic layer structure of a Figure 4: Comparison of measured transsingle heterojunction AlGaN/GaN HEMT fer characteristics and simulations with with field-plate.

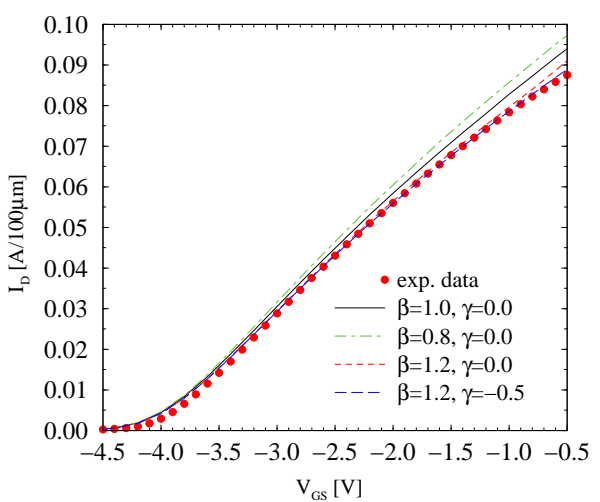

different mobility model parameters.

\section{Simulation Results}

The transfer characteristics at $V_{D S}=12 \mathrm{~V}$ are shown in Fig. 4. Simulation results with four different model parameter sets illustrate the calibration of the new mobility model against the experimental data. As can be seen the parameter set $\beta=1.2$ and $\gamma=-0.5$ gives the best agreement.

Fig. 5 compares velocity-field characteristics, as resulting from hydrodynamic device simulations using the four parameter sets. Bulk and device MC simulation data from various groups are also included. As can be seen in the figure, higher $\beta$ values result in lower peak velocity, while negative $\gamma$ values decrease the velocity at higher electric fields. Fig. 6 shows the electron temperature and drift velocity for two different parameter sets in a cut along the channel below the gate $(x=0.0-0.3 \mu \mathrm{m})$ and the field-plate $(x=0.3-0.6 \mu \mathrm{m})$. The peak electron temperatures are found just below the drain-side edge of the gate $(x=0.3 \mu \mathrm{m})$. While the temperature profiles do not differ significantly, the new model leads to lower velocity, especially in the high electron temperature region.

Using the four parameter sets an AC analysis was performed. Fig. 7 shows experimental and simulated data for the input capacitance $\mathrm{C}_{11}$ and the gate-drain capacitance $\mathrm{C}_{\mathrm{gd}}$. The altered mobility model parameters have some impact only on $\mathrm{C}_{11}$ for higher $\mathrm{V}_{\mathrm{GS}}$ values. Fig. 8 presents the cut-off frequency $f_{\mathrm{T}}$ as a function of gate voltage $\mathrm{V}_{\mathrm{GS}}$ at $\mathrm{V}_{\mathrm{DS}}=12 \mathrm{~V}$. Again, the best agreement between simulation and experimental data is achieved by using the new model and the parameter set $\beta=1.2, \gamma=-0.5$. The simulation results overestimate the measured data by about $20 \%$ because of higher gate-source capacitance $\mathrm{C}_{\mathrm{gs}}$ in the real structure.

\section{Conclusion}

We propose a refined mobility model accounting for the specifics of electron transport in the GaN material system. We explore the effects on DC and AC characteristics of real HEMT structures and achieve a good agreement with experimental data. 

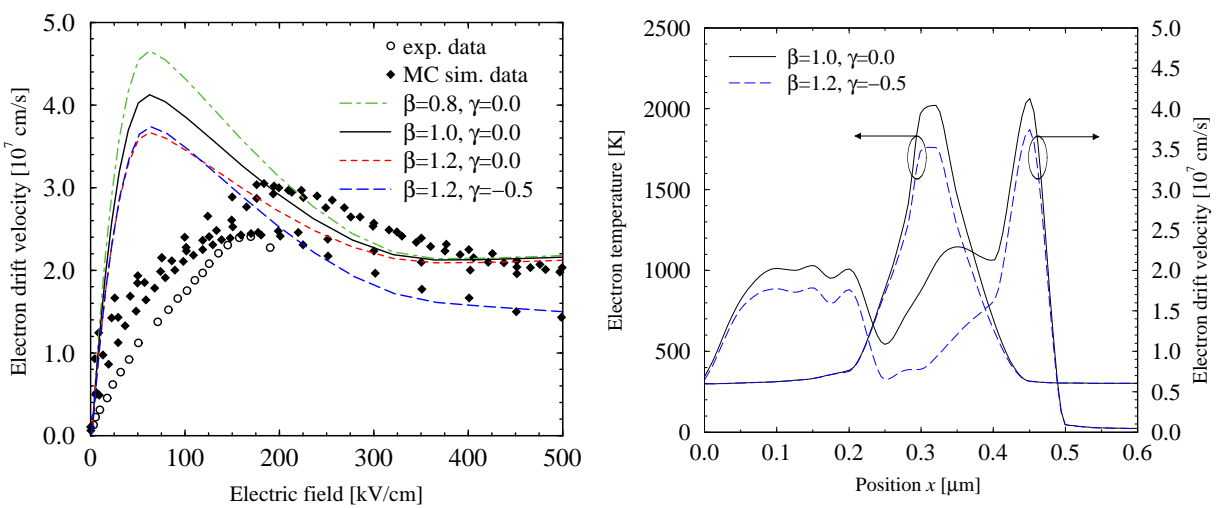

Figure 5: Electron drift velocity versus Figure 6: Simulated electron temperature electric field: simulations with different and drift velocity along the channel of the mobility model parameter values. device at $\mathrm{V}_{\mathrm{DS}}=12 \mathrm{~V}$ and $\mathrm{V}_{\mathrm{GS}}=-4.0 \mathrm{~V}$.
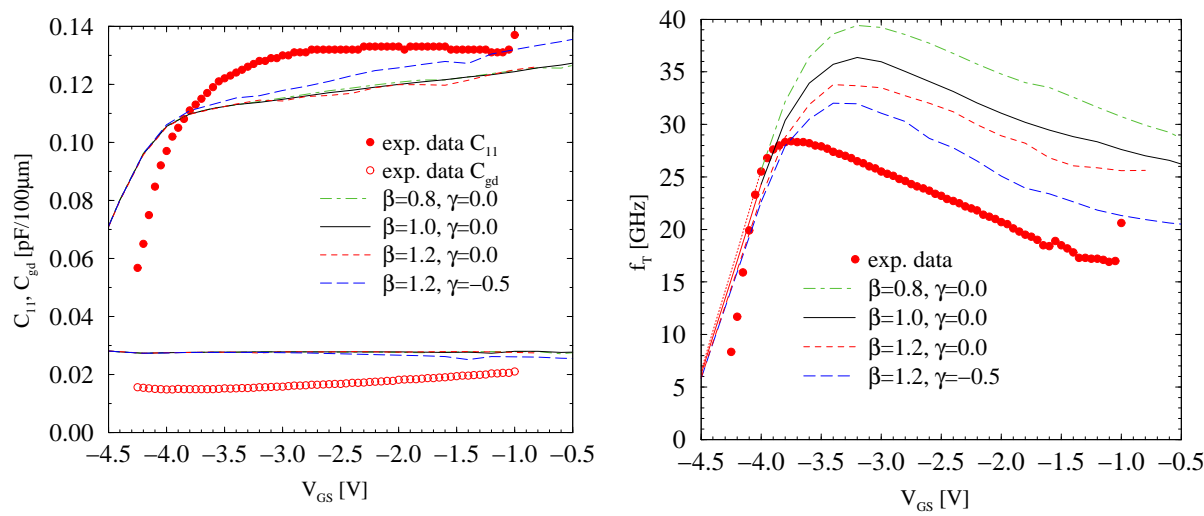

Figure 7: Comparison of measured $C_{11}$ Figure 8: Comparison of measured cutand $\mathrm{C}_{\mathrm{gd}}$ versus $\mathrm{V}_{\mathrm{GS}}$ and simulations with off frequency versus $\mathrm{V}_{\mathrm{GS}}$ and simulations different mobility model parameters. with different mobility model parameters.

\section{Acknowledgments}

Support by the Austrian Science Funds FWF and BMBWK, START Project No.Y247N13, is acknowledged.

\section{References}

[1] Minimos-NT Device and Circuit Simulator, User's Guide, Release 2.0, 2000, http://www.iue.tuwien.ac.at/mmnt.

[2] S. Vitanov, V. Palankovski, R. Quay, and E. Langer, in 28th ICPS, p. 244, 2006.

[3] D. Caughey and R. Thomas, Proc.IEEE, vol. 52, pp. 2192-2193, 1967.

[4] W. Hänsch, M. Orlowski, and W. Weber, in 18th ESSDERC, pp. 597-606, 1988.

[5] V. Palankovski and R. Quay, Analysis and Simulation of Heterostructure Devices. Wien, New York: Springer, 2004. 